\title{
Virus papiloma humano y Chlamydia trachomatis según número de parejas sexuales y tiempo de actividad sexual en estudiantes universitarias en la Región de La Araucanía, Chile
}

\author{
Angélica Melo, Nicole Lagos, Sonia Montenegro, Juan José Orellana, Ana María Vásquez, \\ Sergio Moreno, Sandra Liempi, Pablo Guzmán y Flery Fonseca-Salamanca
}

\begin{abstract}
Human papilloma virus and Chlamydia trachomatis by number of sexual partners and time of sexual activity on university students in the Region of La Araucanía, Chile
\end{abstract}

Background: Human papilloma virus (HPV) and Chlamydia trachomatis are the most prevalent sexually transmitted infections (STIs), among teenagers and young people, with risk factors: active sex life and multiple partners. Chlamydia trachomatis infection may favor HPV infection and this, the development of cervical cancer. Both infections can lead to consequences on sexual and reproductive health. Objective: To determine frequency of HPV and C. trachomatis in asymptomatic university women less than 25 years, associating them with number of sexual partners ( $\left.\mathrm{n}^{\circ} \mathrm{SxP}\right)$ and time of sexual activity (TSxA). Material and Methods: 151 cervical samples for HPV and $C$. trachomatis, were processed by conventional and in real time reaction polymerase chain. Results: HPV 21, 8\%, C. trachomatis 11, 2\% and co-infection (HPV/C.trachomatis), 4.6\%. Among HPV +, 80, 6\% showed high risk HPV. The ${ }^{\circ}{ }^{S} \mathrm{XP}$ was strongly associated with HPV. Among young coinfected HPV/C. trachomatis, $71.4 \%$ had 3 or more PSx. Chlamydia trachomatis was more frequent (64,7\%) that HPV within range of 3-5 years according to the TSxA, Discussion: A high prevalence of HPV and C. trachomatis was observed. Young women with coinfection $\mathrm{HPV} / C$. trachomatis could be a high-risk group need to monitor their infections. It suggests the implementation of university programs in education, counseling and prevention in sexual health.

Key words: HPV, Chlamydia trachomatis, young women, university students, sexual partners, risk factors.

Palabras clave: VPH, Chlamydia trachomatis, mujeres jóvenes, estudiantes universitarias, parejas sexuales, factores de riesgo.

\section{Introducción}

$\mathrm{E}$ 1 virus papiloma humano (VPH) y Chlamydia trachomatis son consideradas las infecciones de transmisión sexual (ITS) más frecuentes en el mundo ${ }^{1,2}$. En mujeres jóvenes con inicio precoz de vida sexual, la prevención, diagnóstico y tratamiento en etapas iniciales de estas infecciones es relevante para su salud sexual y reproductiva y evitar consecuencias graves como enfermedad inflamatoria pélvica, infertilidad y/o cáncer cérvico uterino ${ }^{3-6}$.

Los VPH de alto riesgo oncogénicos (VPHAR) son considerados agentes etiológicos de la carcinogénesis cervical y el principal factor de riesgo de desarrollar neoplasia intraepitelial cervical (NIC) y cáncer cérvico uterino (CCU). Esta progresión se daría bajo ciertas condiciones como: cantidad de virus y tiempo de exposición, persistencia prolongada de virus oncogénicos en el epitelio cervical (auto-infección), como también, la posibilidad de acceder a programas de prevención del $\mathrm{CCU}$ o detección del $\mathrm{VPH}^{7-9}$.
La infección por VPH puede ser adquirida en las jóvenes en los primeros meses después de la primera relación heterosexua ${ }^{10} \mathrm{y}$, aunque la mayoría de las jóvenes elimina en forma natural el virus, se conoce un grupo de mujeres que la infección por VPH carcinogénicos puede persistir, acumulándose un daño genético en la célula hospedera y eventualmente llevar a una progresión de la lesión ${ }^{11,12}$.

Por otro lado, la infección por C. trachomatis puede favorecer el ingreso del VPH al epitelio cervical y ser un co-factor en la etiología del CCU. Se estima que en $20 \%$ de los cánceres podría estar presente C. trachomatis. Su presencia podría dañar los cilios y obstruir las trompas de Falopio, aumentando el riesgo de embarazos ectópicos y provocar infertilidad en algunas mujeres ${ }^{13-15}$.

En países, como Estados Unidos de América, Inglaterra, Suecia y España, se han implementado programas de monitoreo focalizados en el diagnóstico y tratamiento de la población sintomática, así como, la detección de la infección en población asintomática y notificación a la pareja sexual ${ }^{2,16-20}$. En Chile, a partir del año 2007, y debido a la elevada frecuencia de estas ITS, se inició la
Universidad de La Frontera, Temuco, Chile. Facultad de Medicina Departamento Anatomía Patológica (AM, PG).

Departamento de Salud Pública (JJO).

Departamento Obstetricia y Ginecología (AMV). Departamento de Ciencias Preclínicas.Laboratorio de Inmunoparasitología Molecular, CEGIN-BIOREN (AM, NL, FF-S). Universidad de Concepción. Facultad de Medicina. Departamento de Especialidades. Laboratorio de Diagnóstico Clínico Molecular-UDEC (SM). Hospital Hernán Henríquez Aravena de Temuco. Unidad de Anatomía Patológica (SM, SL, PG).

Financiamiento: Proyecto DIUFRO DI 15-0047 y DI 01139 Los autores declaran que no existen conflictos de interés

Recibido: 9 de marzo de 2016 Aceptado: 24 de mayo de 2016

Correspondencia a: Angélica Melo Angermeyer angélica.melo@ufrontera.cl 
vigilancia de la infección por VPH y $C$. trachomatis en centros centinela. Al igual que en otros países, el principal grupo etario afectado son las mujeres bajo 25 años de edad, en quienes predominan los factores de riesgo más importantes: vida sexual activa y parejas múltiples ${ }^{21}$.

En relación a las metodologías usadas para la detección de $C$. trachomatis y VPH, ya sea en programas de cribado o en estudio realizados, tanto en poblaciones asintomáticas como sintomáticas, las más utilizadas por su sensibilidad y especificidad son las metodologías moleculares, principalmente la reacción de polimerasa en cadena (RPC) y otras técnicas como la hibridación reversa, para la genotipificación del $\mathrm{VPH}^{22-25}$.

Considerando que existen mujeres sexualmente activas bajo 25 años de edad no contempladas en el programa nacional de tamizaje para $\mathrm{CCU}$, que la infección por C. trachomatis puede facilitar la entrada del VPH a la mucosa cervical y que en la Región de La Araucanía no existen estudios publicados de prevalencia en estudiantes universitarias, se planteó como objetivo determinar en muestras cervicales en esta población, la presencia de VPH y $C$. trachomatis, mediante la RPC y asociar su presencia con las variables de tiempo de actividad sexual y número de parejas sexuales.

\section{Material y Método}

\section{Pacientes}

Participaron voluntariamente 151 estudiantes universitarias sexualmente activas, asintomáticas, bajo 25 años de edad, que respondieron a una invitación masiva realizada a la comunidad universitaria mediante afiches y correos electrónicos, para tomarse el examen de Papanicolaou (Pap) sin costo durante el año 2013 y 2014. Previo a la toma de muestra cervical se obtuvo un consentimiento informado aprobado por el Comité de Ética Científica de la Universidad de La Frontera. El registro de la información se realizó mediante un formulario adaptado de la $7^{\text {a }}$ Encuesta Nacional de la Juventud en lo que respecta a: primera relación sexual y $\mathrm{n}^{0}$ de parejas sexuales ${ }^{26}$. Estos puntos fueron respondidos en forma confidencial por cada participante. Para este estudio se consideraron los antecedentes: edad, tiempo de actividad sexual (años entre la edad de la primera relación sexual y la edad al momento del estudio) y $\mathrm{n}^{\circ}$ de parejas sexuales en los últimos tres años.

\section{Muestras clínicas}

Se obtuvieron muestras cervicales mediante un cepillado (citobrush) de la zona exo-endocervical. El citobrush fue colocado en $3 \mathrm{ml}$ de tampón fosfato salino (PBS1X), trasladado al laboratorio refrigerado y procesado dentro de $24 \mathrm{~h}$. El concentrado de células cervicales exfoliadas fue tratado con solución de lisis (kit E.Z.N.A. ${ }^{\circledR}$ Tissue DNA) y almacenado a $-80{ }^{\circ} \mathrm{C}$ hasta su procesamiento. Las muestras fueron procesadas en el Laboratorio de Inmunoparasitología Molecular-CEGIN y la tipificación del VPH se realizó en el laboratorio de diagnóstico clínico molecular-UDEC.

\section{Extracción del ADN}

Se utilizó el kit E.Z.N.A.® Tissue DNA (Omega BioTek USA), siguiendo las instrucciones del fabricante. Control interno: Para comprobar la integridad del ADN obtenido y descartar muestras de ADN no amplificable, se realizó una RPC con los partidores PCO4 5'-CAACTTCATCCA CGTTCACC-3' y GH20 5'-GAAGAGCCAAGGACAGGTAC-3' que amplifican un fragmento de 268 pb del gen de la Beta-globina ${ }^{24}$.

\section{Detección del VPH}

Para la detección del VPH se utilizó una RPC anidada (RCPL1) de secuencias del gen L1 que amplifica un fragmento de $150 \mathrm{pb}$ empleando los partidores externos MY11 y MY09 e internos GP5+/GP6 $+{ }^{25}$. La concentración de los partidores fue de $0,45 \mu \mathrm{M}$ y el volumen de reacción de $15 \mu$ l. El perfil térmico fue: $1^{\text {a }}$ amplificación 30 ciclos, $94{ }^{\circ} \mathrm{C}$ por $30 \mathrm{seg}, 45^{\circ} \mathrm{C}$ por $1 \mathrm{~min}, 72^{\circ} \mathrm{C}$ por $1 \mathrm{~min} ; 2^{\mathrm{a}}$ amplificación 40 ciclos, $94^{\circ} \mathrm{C}$ por $30 \mathrm{seg}, 40^{\circ} \mathrm{C}$ por $1 \mathrm{~min}$, $72^{\circ} \mathrm{C}$ por $1 \mathrm{~min}$. La banda de $150 \mathrm{pb}$ que se observó luego de electroforesis, indicó presencia de VPH.

\section{Tipificación del VPH}

Para diferenciar entre VPH AR y VPH de bajo riesgo (BR) se empleó una RPC en tiempo real (Maxima SYBR Green/ROX qRCP, Thermo Fisher) utilizando partidores dirigidos a los genes E6/E7 del VPH. Las secuencias de los partidores fueron las siguientes: $A R$ PU1M: 5'-TGTCAAAAACCGTTGTGTCC-3', BR PU31B: 5'-TGYTAATTCGGTGYTACCTG-3' y ARBR PU2R: 5'-GAGCTGTCGCTTAATTGCTC-3'27. La concentración de los partidores fue de $1 \mu \mathrm{M}$ y el volumen de reacción de $13 \mu 1$. Perfil térmico: $95^{\circ} \mathrm{C}$ por $10 \mathrm{~min}, 40$ ciclos de $94{ }^{\circ} \mathrm{C}$ por $20 \mathrm{seg}, 40{ }^{\circ} \mathrm{C}$ por $25 \mathrm{seg}$ y $72{ }^{\circ} \mathrm{C}$ por $30 \mathrm{seg}$. Como controles positivos se emplearon plásmidos comerciales (ATCC) para VPH 6, 11, 16 y 18.

\section{Detección de C. trachomatis}

Se realizó mediante una RPC simple (RCPCT) utilizando los partidores KL1 5'-TCCGGAGCGAGTTACGAAGA-3'KL25'-AATCAATGCCCGGGATTGGT-3' ${ }^{28}$ que amplifican un fragmento de $241 \mathrm{pb}$ del plásmido críptico de $C$. trachomatis. Perfil térmico: 35 ciclos, denaturación: $94{ }^{\circ} \mathrm{C}$ por $30 \mathrm{seg}$, hibridación: $55^{\circ} \mathrm{C}$ por $30 \mathrm{seg}$, extensión $72{ }^{\circ} \mathrm{C}$ por 30 seg. La banda de 241 pb que se observa luego de electroforesis, indicó presencia de C. trachomatis. Como controles positivos se emplearon muestras clínicas 
conocidas positivas para $C$. trachomatis diagnosticadas mediante el kit comercial para RPC en tiempo real (Abbott RealTime CT/NG assay).

\section{Visualización}

Los productos RPC mezclados con tampón de carga y gel red fueron cargados en geles de agarosa al $2 \%$ y corridos a 100 volts durante 45 min y observados en transiluminador.

\section{Análisis de resultados}

Los resultados de cada participante fueron identificados con un código y vaciados a una base digital, junto a los datos recabados en la encuesta inicial. Se realizó un análisis descriptivo utilizando tablas de contingencia con frecuencias absolutas y relativas. Los datos de las variables cuantitativas son resumidos con los estadígrafos rango, promedios y desviaciones estándar.

\section{Resulltados}

El grupo de estudio estuvo compuesto por 151 estudiantes universitarias con edades de 18 a 24 años cumplidos, con edad promedio de 21,5 años. El número de parejas sexuales (n ${ }^{\circ} \mathrm{PSx}$ ) en los últimos tres años fue: una pareja $39,1 \%$, dos parejas $33,8 \%$ y tres o más parejas $27,1 \%$. El tiempo de actividad sexual (TASx) fue: menor a tres años en $31,8 \%$, tres a cinco años en $44,4 \%$ y seis a ocho años en $23,8 \%$.

Del total estudiado, 57 (37,7\%) jóvenes presentaron algún tipo de infección: 33 (21,8\%) fueron infectadas por VPH, $17(11,2 \%)$ por C. trachomatis y en $7(4,6 \%)$ se observó co-infección.

En la Tabla 1 se destaca que la frecuencia de infección por VPH aumentó proporcionalmente según el $\mathrm{n}^{\mathrm{o}}$ de parejas sexuales: 15,1\% (una pareja), 33,3\% (dos parejas) y $51,5 \%$ (tres o más parejas), hecho que no se presentó en los casos de infección por C. trachomatis. En relación al TASx, en el rango 3-5 años se observó 33,3\% de infección por VHP y $64,7 \%$ de jóvenes infectadas con C. trachomatis. En el grupo con co-infección (VPH/C. trachomatis), 71,4\% presentó tres o más parejas sexuales y $57,1 \%$ tenía entre tres y cinco años de actividad sexual.

Un 29,2\%(7/24) de mujeres con infección por $C$. trachomatis tenía infección por VPH; en cambio, 17,5\% (7/40) de mujeres con infección por VPH presentaron co-infección con C. trachomatis.

La clasificación de los casos de infección por VPHAR y VPH de bajo riesgo (VPHBR) oncogénico fue realizada mediante la RCPE6/E7 en tiempo real, se consideraron los 33 casos infectados con VPH y los siete casos con co-infección por VPH y C. trachomatis. En cuatro de estos 40 casos no fue posible genotipificar estas muestras quedando como no clasificables. Dos casos que presen-

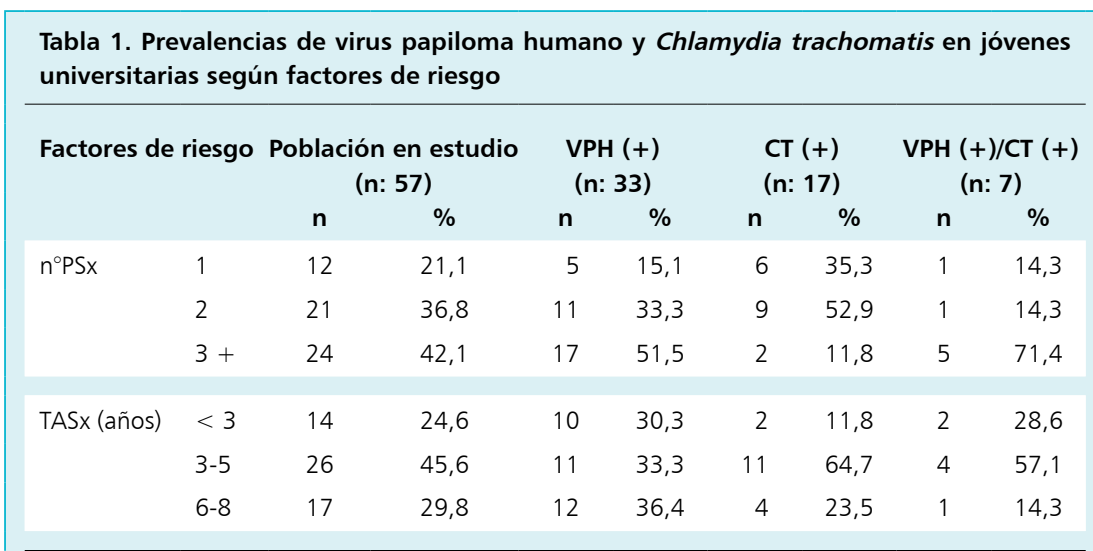

TASx (años cumplidos): Tiempo de actividad sexual. noPSx: número de parejas sexuales. VPH (+): VPH positivo. CT (+): Chlamydia trachomatis positiva. VPH (+)/CT (+): co-infección.

Tabla 2. Tipificación del VPH en alto riesgo y bajo riesgo en el grupo de jóvenes con infección por VPH según factores de riesgo

\begin{tabular}{|c|c|c|c|c|c|c|c|}
\hline \multicolumn{2}{|c|}{ Factores de riesgo } & \multicolumn{6}{|c|}{ Tipificación VPH } \\
\hline & & \multicolumn{2}{|c|}{$\begin{array}{l}\text { Población con VPH+ } \\
(\mathrm{n}: 36)^{*}\end{array}$} & \multicolumn{2}{|c|}{$\begin{array}{c}\text { AR } \\
(n: 29)\end{array}$} & \multicolumn{2}{|c|}{$\begin{array}{l}\text { BR } \\
(n: 7)\end{array}$} \\
\hline & & $\mathrm{n}$ & $\%$ & $\mathbf{n}$ & $\%$ & $\mathrm{n}$ & $\%$ \\
\hline \multirow[t]{3}{*}{$n^{\circ} P S x$} & 1 & 5 & 13,9 & 4 & 80,0 & 1 & 20,0 \\
\hline & 2 & 10 & 27,8 & 10 & 100,0 & 0 & 0,0 \\
\hline & $3+$ & 21 & 58,3 & 15 & 71,4 & 6 & 28,6 \\
\hline \multirow[t]{3}{*}{ TASx (años) } & $<3$ & 11 & 30,6 & 9 & 81,8 & 2 & 18,2 \\
\hline & $3-5$ & 13 & 36,1 & 12 & 92,3 & 1 & 7,7 \\
\hline & $6-8$ & 12 & 33,3 & 8 & 66,7 & 4 & 33,4 \\
\hline
\end{tabular}

taron co-infección $\mathrm{AR} / \mathrm{BR}$ se incorporaron al grupo de AR. En 80,6\% (29/36) de las jóvenes se detectó VPHAR, observándose 19,4\% (7/36) con VPHBR.

Las jóvenes con tres o más parejas presentaron $71,4 \%$ de VPHAR y $28,6 \%$ de VPHBR. Al comparar la presencia de virus alto y bajo riesgo oncogénico con el TASx, 92,3\% eran jóvenes VPHAR y con 3-5 años de actividad sexual (Tabla 2).

En el grupo con co-infección VPH/C. trachomatis, cinco de siete jóvenes presentaron VPHAR, las cuales habían tenido cuatro o más parejas sexuales en los últimos tres años y sobre cuatro años de actividad sexual.

\section{Discusión}

El presente estudio es el primero en la Región de La Araucanía que reporta la frecuencia de VPH/C. trachomatis y su asociación con el número de parejas sexuales y 
el tiempo de actividad sexual en estudiantes universitarias bajo 25 años de edad.

El grupo, tuvo en promedio 17,7 años de edad al iniciar relaciones sexuales y 1,8 parejas sexuales (datos no mostrados en los resultados) y, si bien el diseño de reclutamiento pudiese introducir un sesgo como efecto de la selección, éste probablemente no sea significativo, dado la similitud con lo reportado en jóvenes chilenos ${ }^{26}$.

Ambas ITS están presentes en este grupo de estudio con una alta prevalencia que es importante considerar. Desde el punto de vista de salud pública, esto implica, que se trata de un grupo potencialmente vulnerable para el desarrollo de lesiones del aparato reproductivo, de enfermedad inflamatoria pélvica o infertilidad y eventualmente un cáncer cervical precoz. En un estudio anterior sobre genotipificación del VPH, realizado en mujeres bajo 25 años de edad, tratadas en la Unidad de Patología Cervical, se observó que las jóvenes habían comenzado a desarrollar lesiones antes de los 25 años, desde atipias celulares hasta NIC, que en el futuro pudieran traducirse en un cáncer cervical ${ }^{24}$

La prevalencia de VPH $(21,8 \%)$ encontrada en el presente estudio fue similar a reportes previos. Almonte y cols., mencionan que la prevalencia para VPH en América Latina bordea los 25 a $30 \%$ en mujeres jóvenes ${ }^{29}$. En Chile, se obtuvo una prevalencia de $30,9 \%$ de VPH en muestras cérvico-vaginal de mujeres bajo 25 años de edad, asintomáticas ${ }^{30}$. En el concierto internacional se reportan para adolescentes y mujeres jóvenes una frecuencia de VPH de $35 \%$ en muestras de vulva-vagina y $24,2 \%$ en muestras cervicales ${ }^{1,31}$.

En relación a los factores de riesgos evaluados, más de la mitad $(58,3 \%)$ de las jóvenes con infección por VPH declaró haber tenido tres o más parejas en los últimos tres años. Un aspecto a destacar aquí, es que la mayoría de estas jóvenes presentaron genotipos VPH de alto riesgo $(71,4 \%)$. En reportes previos entre el VPH y el n ${ }^{\circ} \mathrm{PSX}$ se ha encontrado una asociación significativa además, entre el VPH y el inicio sexual antes de los 15 años ${ }^{1}$. En relación a esto último, el $\mathrm{n}^{\mathrm{o}}$ de casos con infección por VPH fue similar según los rangos de TASx establecidos en el presente estudio, lo que indicaría que esta variable no sería un factor de riesgo evidente. Por otro lado, 48,5\% de las jóvenes infectadas por VPH tenían entre una y dos parejas. Al parecer, la mantención de pareja única no descarta que las jóvenes lleguen a infectarse, si su pareja desconoce ser un portador del virus y mantienen relaciones sexuales sin preservativo; el hombre, como reservorio del VPH, ayudará a que la infección persista en su pareja. Un estudio previo realizado en la región reveló altas cifras de infección por VPH en estudiantes universitarios masculinos; posiblemente, algunos de estos jóvenes pueden haber sido parejas de las jóvenes actualmente en estudio ${ }^{32}$.
Con respecto a la prevalencia de $C$. trachomatis obtenida $(11,2 \%)$, fue superior a la observada en un estudio de jóvenes catalanas entre 16 y $24 \operatorname{años}^{33}$ y a la reportada en adolescentes de Santiago de Chile $(6,9 \%)^{34}$. Sin embargo, fue similar al estudio de Silva y cols., en mujeres de 15-64 años realizado en la Región de La Araucanía (11,49\%) y al de Magalhaes y cols., en mujeres brasileñas entre 14-64 años en la Región de Río Grande $(10,9 \%)^{35,36}$.

En relación a las jóvenes infectadas por C. trachomatis y $n^{\circ} P S x$, Hunneus y cols., reportaron que esta variable no fue considerada como un factor de riesgo ${ }^{34}$; del mismo modo, se podría inferir de los datos obtenidos en el presente estudio, donde no se observa un aumento franco a mayor $\mathrm{n}^{\circ} \mathrm{PSx}$. En relación al TASx, un alto porcentaje $(64,7 \%)$ de las jóvenes con C. trachomatis se observan en el rango de 3-5 años. Una explicación a ello podría ser que en este rango se concentran las jóvenes con tres o más parejas sexuales, y no así, en los rangos de menos de tres años y seis a ocho años de actividad sexual. De hecho, Corbeto y cols., mencionan como factor de riesgo independiente para la infección por C. trachomatis el tener una nueva pareja sexual en un corto período de tiempo ${ }^{33}$; también hacen referencia a ello y al inicio precoz de actividad sexual Magalhaes y cols ${ }^{36}$. Desde este punto de vista, el $n^{\circ} \mathrm{PSx}$ estaría mostrando en forma indirecta la probabilidad que tienen las jóvenes universitarias de infectarse con el VPH como también con C. trachomatis.

En América Latina, un estudio realizado en mujeres aborígenes de la comunidad Pilaga, detectó prevalencias de $46,7 \%$ para VPH, 26,4\% para C. trachomatis y $16,3 \%$ para la co-infección VPH/C. trachomatis ${ }^{15}$. En la región europea, un estudio reciente en jóvenes italianas reportó prevalencias de $18,2,5,8$ y $2,7 \%$ para $\mathrm{VPH}, C$. trachomatis y co-infección, respectivamente ${ }^{37}$, más bajas que las reportadas en el presente estudio. Las diferencias observadas en las prevalencias, claramente se han visto influenciadas por la edad, factores socio-culturales, étnicos y geográficos de las poblaciones estudiadas ${ }^{38}$.

En relación a la co-infección, se puede destacar que hubo una mayor probabilidad de encontrar co-infección con VPH en las jóvenes con C. trachomatis. A 29,2\% de las jóvenes infectadas con $C$. trachomatis se les detectó VPH. Esto está apoyando lo reportado sobre $C$. trachomatis, que sería un co-factor para infección por $\mathrm{VPH}^{14-16}$.

Del estudio de Magalhaes y cols., cabe señalar que la mayoría de la mujeres con infección por C. trachomatis tenía menos de 32 años y la prevalencia de $C$. trachomatis fue de $10,0 \%$ en las mujeres con un examen citológico de Papanicolaou (Pap) normal ${ }^{36}$. En relación al grupo de estudio, las jóvenes universitarias con $C$. trachomatis al momento de la toma de muestra cervical no presentaban síntomas. Sin embargo, 4/24 (datos no mostrados en los resultados) presentaron un Pap anormal (Pap atípico y NIC Grado I) y fueron positivas para VPH. En este as- 
pecto, las jóvenes con co-infección (4,6\%) en el presente estudio pueden constituir un grupo de alto riesgo, donde cobra mucha importancia el tipo de VPH (la mayoría con VPHAR) y la presencia de $C$. trachomatis que, en forma silenciosa, puede dañar la mucosa aumentando el riesgo de re-infección o incluso, interferir en la respuesta inmune y en la eliminación del VPH permitiendo que persista la infección ${ }^{13,39}$.

Los cuatro casos de infección por VPH que no fueron clasificados en AR o BR, pudieran deberse a que la amplificación del gen L1 es más sensible que la de los oncogenes E6/E7. Además, las muestras fueron procesadas con un intervalo de tiempo de casi un año, por lo que pudo haber algún grado de degradación del ADN.

Como es sabido, la infección del VPH se ha descrito que se adquiere predominantemente en la adolescencia con un pico en la mujer joven menor de 25 años. Aunque se ha mencionado que la detección del VPH en mujeres jóvenes sería de poca utilidad clínica ${ }^{11}$, la detección del VPH en jóvenes universitarias que aún no ingresan al programa de tamizaje para CCU, adquiere importancia pues se podría identificar un grupo de jóvenes con infección persistente, sobre todo en esta población que tiende a interrelacionarse dentro de un ámbito cerrado y que además puede tener factores de riesgo adjunto, como múltiples parejas sexuales, u otra ITS como $C$. trachomatis, que siendo una ITS de fácil tratamiento, sino es tratada a tiempo, puede a futuro afectar la salud reproductiva de las jóvenes.

En conclusión, el estudio detectó altas frecuencias de VPH y C. trachomatis y pone un foco de atención en estas infecciones que se presentan asintomáticas. Además identificó a jóvenes con co-infección VPH/C. trachomatis, grupo potencialmente vulnerable, que podría considerarse de alto riesgo y necesitado de monitoreo para evitar eventuales lesiones y secuelas en su aparato reproductor.

Finalmente, sin desconocer las recomendaciones del programa nacional de tamizaje del cáncer cérvico uterino, los resultados de este estudio sugieren que sería beneficioso implementar y aplicar dentro del ámbito universitario programas de educación, orientación y prevención en ITS, pre-ingreso de estas jóvenes, al programa de tamizaje del CCU y por qué no a futuro, a un programa nacional para el diagnóstico de rutina de $C$. trachomatis en adolescentes y jóvenes.

\section{Resumen}

Introducción: El virus papiloma humano (VPH) y Chlamydia trachomatis son las infecciones de transmisión sexual (ITS) más frecuentes, en adolescentes y jóvenes, con factores de riesgo: vida sexual activa y múltiples parejas. Chlamydia trachomatis puede favorecer el ingreso de VPH y éste el desarrollo del cáncer cérvico uterino. Ambas infecciones pueden dejar secuelas en la salud sexual y reproductiva. Objetivo: Determinar frecuencias VPH y $\mathrm{C}$ trachomatis en estudiantes universitarias asintomáticas bajo 25 años, asociándolas con número de parejas sexuales ( $\left.{ }^{\circ} \mathrm{PSx}\right)$ y tiempo de actividad sexual (TASx). Material y Método: Se procesaron 151 muestras/exo y endo cervicales para VPH y C. trachomatis, mediante reacción de polimerasa en cadena convencional y en tiempo real. Resultados: La frecuencia fue: VPH 21,8\%, C. trachomatis $11,2 \%$ y co-infección 4,6\%. De las jóvenes con infección por $\mathrm{VPH}, 80,6 \%$ presentó $\mathrm{VPH}$ alto riesgo oncogénico. El ${ }^{\circ} \mathrm{PSx}$ se asoció fuertemente a VPH. Entre las jóvenes con co-infección VPH/C. trachomatis, 71,4\% tenían tres o más PSx. Según TASx, C. trachomatis fue más frecuente $(64,7 \%)$ entre 3-5 años que VPH. Conclusión: Se observó alta prevalencia de VPH y C trachomatis. Mujeres jóvenes con co-infección VPH/C. trachomatis podrían ser un grupo de alto riesgo con necesidad de monitorear sus infecciones. Es sugerida la implementación de programas universitarios en educación, orientación y prevención en ITS.

\section{Referencias bibliográficas}

1.- Roset Bahmanyar E, Paavonen J, Naud P, Salmeron J, Chow S N, Apter D, et al. Prevalence and risk factors for cervical HPV infection and abnormalities in young adult women at enrolment in the multinational PATRICIA trial. Gynecol Oncol 2012; 127 (3): 440-50.

2.- Centers for Disease Control and Prevention. Sexually transmitted disease surveillance 2013. Atlanta: US Department of Health and Human Services 2014.

3.- Baquedano L, Lamarca M, Puig F, Ruiz M A. Enfermedad inflamatoria pélvica: un reto en el diagnóstico y tratamiento precoz. Rev Chil
Obstet Ginecol 2014; 79 (2): 115-20.

4.- Satterwhite C L, Chow J M, Bernstein K T, Guerry S L, Nakatsukasa-Ono W, Bauer H M. Opportunities for Chlamydia control in the era of healthcare reform: lessons from two decades of innovative family planning care. Womens Health (Lond Engl) 2013; 9 (1): 25-38.

5.- Haggerty C L, Gottlieb S L, Taylor B D, Low N, Xu F, Ness R B. Risk of sequelae after Chlamydia trachomatis genital infection in women. J Infect Dis 2010; 201 Suppl 2: S134-55.

6.- Schiffman M, Wentzensen N, Wacholder S, Kinney W, Gage J C, Castle P E. Human papillomavirus testing in the prevention of cervical cancer. J Natl Cancer Inst 2011; 103
(5): 368-83.

7.- Kjaer S K, Frederiksen K, Munk C, Iftner T. Long-term absolute risk of cervical intraepithelial neoplasia grade 3 or worse following human papillomavirus infection: role of persistence. J Natl Cancer Inst 2010; 102 (19): 1478-88.

8.- Plummer M, Peto J, Franceschi S. Time since first sexual intercourse and the risk of cervical cancer. Int J Cancer 2012; 130 (11): 2638-44

9.- MINSAL. Guía Clínica Cáncer Cervicouterino. Salud Md, (ed). Consultado 25 de noviembre de 2015. Disponible en: http://www.minsal.cl/ portal/url/item/720bfefe91e9d2ede04001011f01 0ff2.pdf 2010. 
10.- Winer R L, Feng Q, Hughes J P, O'Reilly S, Kiviat N B, Koutsky L A. Risk of female human papillomavirus acquisition associated with first male sex partner. J Infect Dis 2008; 197 (2): 279-82.

11.- Moscicki A B, Ma Y, Jonte J, MillerBenningfield S, Hanson E, Jay J, et al. The role of sexual behavior and human papillomavirus persistence in predicting repeated infections with new human papillomavirus types. Cancer Epidemiol Biomarkers Prev 2010; 19 (8): 2055-65.

12.- Doorbar J, Quint W, Banks L, Bravo I G, Stoler M, Broker T R, et al. The biology and life-cycle of human papillomaviruses. Vaccine 2012; 30 Suppl 5: F55-70.

13.- Silva J, Cerqueira F, Medeiros R. Chlamydia trachomatis infection: implications for HPV status and cervical cancer. Arch Gynecol Obstet 2014; 289 (4): 715-23.

14.- Frontela M, Rodríguez Y, Ríos M, Hernández M. Infección por Chlamydia trachomatis como cofactor en la etiología del cáncer cervical. Rev Cubana Obstet Ginecol 2014; 40 (1): 68-78.

15.- Deluca G D, Basiletti J, Schelover E, Vásquez N D, Alonso J M, Marín H M, et al. Chlamydia trachomatis as a probable cofactor in human papillomavirus infection in aboriginal women from northeastern Argentina. Braz J Infect Dis 2011; 15 (6): 567-72.

16.- Red Nacional de Vigilancia Epidemiológica. Instituto de Salud Carlos III. Resultados de la Vigilancia Epidemiológica de las Enfermedades Transmisibles. Informe Final. Disponible en: http://gesdoc.isciii.es/gesdoccontroller?action =download\&id=29/01/2015-f0855cb161 2012 . (Consultado el 5 de noviembre de 2015).

17.- European Centre for Disease Prevention and Control. Sexually transmitted infections in Europe, 1990-2009. Stockholm: ECDC Disponible en: http://ecdc.europa.eu/en/ publications/Publications/110526_SUR_STI_ in_Europe_1990-2009.pdf 2011; 2 Chapter Chlamydia pages 11-27 (Consultado el 5 de noviembre de 2015).

18.- Hocking J S, Walker J, Regan D, Chen M Y, Fairley C K. Chlamydia screening-Australia should strive to achieve what others have not. Med J Aust 2008; 188 (2): 106-8.

19.- Bracebridge S, Bachmann M O, Ramkhelawon K, Woolnough A. Evaluation of a systematic postal screening and treatment service for genital Chlamydia trachomatis, with remote clinic access via the internet: a cross-sectional study, East of England. Sex Transm Infect 2012; 88 (5): $375-81$.

20.- Centers for Disease Control and Prevention.
STDs in adolescents and young adults. Disponible en: http://www.cdc.gov/std/stats13/ adol.htm 2013. (Consultado el 6 de noviembre de 2015).

21.- Santander E, Fich F, Salvo A, Pacheco G, Mendoza MI, Garcés C, et al. Normas de manejo y tratamiento de las Infecciones de Transmisión Sexual (ITS). Primera parte. Rev Chilena Infectol 2009; 26 (2): 174-90.

22.- De la Fuente-Villarreal D, Guzmán-López S, Barboza-Quintana O, González-Ramírez A. Biología del virus del papiloma humano y técnicas de diagnóstico Medicina Universitaria 2010; 12 (49): 231-8.

23.- Marcone V, Recine N, Gallinelli C, Nicosia R, Lichtner M, Degener A M, et al. Epidemiology of Chlamydia trachomatis endocervical infection in a previously unscreened population in Rome, Italy, 2000 to 2009. Euro Surveill 2012; 17 (25)

24.- Melo A, Vásquez A M, Andana A, Matamala M, Pino T, Guzmán P, et al. Genotyping of human papillomavirus in women under 25 years old treated in the screening program for cervical cancer. Rev Chilena Infectol 2014; 31 (5): 542-8.

25.- Meneses E, Muniz M, Mota A, Viera E, Casimiro A, Moura de Melo Cea. HPV detection using primers MY09/MY11 and GP5+/GP6+ in patients with cytologic and/or colposcopic changes. J Bras Patol Med Lab 2014; 50 (4): 280-5.

26.- Instituto Nacional de la Juventud Gobierno de Chile. Séptima Encuesta Nacional de Juventud Consultado el 25 de enero de 2016. Disponible en: http://www.injuv.gob.cl/portal/wp-content/ files_mf/septimaencuestanacionaljuventud2.pdf 2012.

27.- Noda T, Sasagawa T, Dong Y, Fuse H, Namiki M, Inoue M. Detection of human papillomavirus (HPV) DNA in archival specimens of benign prostatic hyperplasia and prostatic cancer using a highly sensitive nested PCR method. Urol Res 1998; 26 (3): 165-9.

28.- Sánchez V, Torres Mata A, Villalba J. Diagnóstico de infección por Chlamydia trachomatis mediante PCR en pacientes que acuden a la Clínica de Especialidades de la Mujer de la Secretaría de la Defensa Nacional. Ginecol Obstet Mex 2009; 77 (1): 13-8.

29.- Almonte M, Murillo R, Sánchez G I, Jerónimo J, Salmeron J, Ferreccio C, et al. New paradigms and challenges in cervical cancer prevention and control in Latin America. Salud Publica Mex 2010; 52 (6): 544-59.

30.- Castillo M, Castillo C, Aravena M. Sistematización de la información sobre cáncer cérvico uterino en Chile: Revisión y análisis de estudios de costo-efectividad de la vacuna contra VPH. Departamento de Economía de la Salud División de Planificación Sanitaria Subsecretaría de Salud Pública MINSAL 2011

31.- Howell-Jones R, de Silva N, Akpan M, Oakeshott P, Carder C, Coupland L, et al. Prevalence of human papillomavirus (HPV) infections in sexually active adolescents and young women in England, prior to widespread HPV immunisation. Vaccine 2012; 30 (26): 3867-75.

32.- Guzmán P, Ili C, Rifo P, Briceño G, Araya J, Villaseca M, et al. Prevalence of human papillomavirus genital infection among male university students. Rev Med Chile 2008; 136 (11): 1381-9.

33.- Corbeto E, Lugo R, Martrób E, Falguerae G, Rosf R, Avecillag A, et al. Prevalencia de la infección por C. trachomatis y $N$. gonhorroeae y determinantes para su adquisición en jóvenes y adultos-jóvenes en Cataluña. Enferm Infecc Microbiol Clin 2011; 29 (2): 96-101.

34.- Huneeus A, Pumarino M G, Schilling A, Robledo P, Bofil M. Rates of Chlamydia trachomatis and Neisseria gonorrhoeae in Chilean adolescents. Rev Med Chile 2009; 137 (12): 1569-74.

35.- Silva R, León D, Viscarra T, Ili C, Roa J C, Sánchez R, et al. Frequency of Chlamydia trachomatis infection in a group of women from Region of Araucania, Chile. Rev Chilena Infectol 2013; 30 (6): 611-5.

36.- Magalhaes P A, Miranda C A, Lima E G, Moizeis R N, de Lima D B, Cobucci R N, et al. Genital tract infection with Chlamydia trachomatis in women attended at a cervical cancer screening program in Northeastern from Brazil. Arch Gynecol Obstet 2015; 291 (5): 1095-102.

37.- Panatto D, Amicizia D, Bianchi $S$, Frati E R, Zotti C M, Lai P L, et al. Chlamydia trachomatis prevalence and chlamydial/HPV co-infection among HPVunvaccinated young Italian females with normal cytology. Hum Vaccin Immunother 2015; 11 (1): 270-6.

38.- Crichton J, Hickman M, Campbell R, Batista-Ferrer H, Macleod J. Socioeconomic factors and other sources of variation in the prevalence of genital Chlamydia infections: A systematic review and meta-analysis. BMC Public Health 2015; 15: 729.

39.- Vriend H J, Bogaards J A, van Bergen J E, Brink A A, van den Broek I V, Hoebe C J, et al. Incidence and persistence of carcinogenic genital human papillomavirus infections in young women with or without Chlamydia trachomatis co-infection. Cancer Med 2015; 4 (10): 1589-98. 